\title{
Haptic perception of linear extent
}

\author{
LAURA ARMSTRONG and LAWRENCE E. MARKS \\ John B. Pierce Laboratory and Yale University, New Haven, Connecticut
}

\begin{abstract}
The perception of linear extent in haptic touch appears to be anisotropic, in that haptically perceived extents can depend on the spatial orientation and location of the object and, thus, on the direction of exploratory motion. Experiments 1 and 2 quantified how the haptic perception of linear extent depended on the type of motion (radial or tangential to the body) when subjects explored different stimulus objects (raised lines or solid blocks) varying in length and in relative spatial location. Relatively narrow, shallow, raised lines were judged to be longer, by magnitude estimation, than solid blocks. Consistent with earlier reports, stimuli explored with radial arm motions were judged to be longer than identical stimuli explored with tangential motions; this difference did not depend consistently on the lateral position of the stimulus object, the direction of movement (toward or away from the body), or the distance of the hand from the body but did depend slightly on the angular position of the shoulder. Experiment 3 showed that the radial-tangential effect could be explained by temporal differences in exploratory movements, implying that the apparent anisotropy is not intrinsic to the structure of haptic space.
\end{abstract}

How do people perceive and judge linear extentslengths or distances that vary along a single spatial dimension-when these are apprehended through selfinitiated actions in manipulatory space? Lederman, Klatzky, Collins, and Wardell (1987) define manipulatory space as the small-scale space that is explored haptically with the arm. Although quantitative studies of the perception of linear extent in manipulatory space go back more than a century (e.g., Jastrow, 1886), some basic issues still are not fully resolved.

One of these concerns the isotropy of perceptual space: How uniform is perceptual space over its several possible axes? Anisotropy seems to be the rule rather than the exception in perceptual systems - and, presumably, arises from potent perceptual mechanisms, given the failure of experience to "correct" the discrepancies. Both vision and passive touch, for instance, have long been known to exhibit systematic deviations from uniformity. E. H. Weber (1834) was apparently the first to note that passive, tactile two-point spatial sensitivity depends systematically on the axis defined by the punctate stimuli. On the arm, for instance, the threshold for detecting spatial separation is smaller when the points are displaced transversely than when they are displaced longitudinally. Thermal spatial acuity varies in a similar manner (Lee, McGillis, \& Greenspan, 1996). Supraliminally, two

This research was supported by Grants DC00818 and DC00271 to L.E.M. from the National Institute on Deafness and Other Communication Disorders of the NIH. Katharine Clemens provided invaluable assistance in the conduct and analysis of Experiment 3, a preliminary version of which was run by Stanley Gartska of The Foote School with the help of Dawn Uttaro; we thank all of them. Correspondence concerning this article should be addressed to L. E. Marks, John B. Pierce Laboratory, 290 Congress Avenue, New Haven, CN 06519 (e-mail: marks@jbpierce.org).

-Accepted by previous editor, Myron L. Braunstein points are perceived to lie farther apart when they fall on the transverse axis than when they fall on the longitudinal axis (Green, 1982), and stimulating the skin electrically, which probably bypasses the tactile receptors and activates nerve fibers directly (see, e.g., Rollman, 1974), produces a similar anisotropy (Marks et al., 1982). Although anisotropy in passive touch has been attributed to dermatomal organization and the shapes of tactile receptive fields (e.g., Green, 1982; Lee et al., 1996), it is also consistent with the notion that spatial discriminability and perceived extent are relationally determined by the shape of the sensory surface, the arm being longer than it is wide. This account is comparable with the hypothesis of Künnapas $(1955,1957)$ that horizontal extents are judged to be smaller than vertical extents in vision, as will be discussed below, because the visual field is wider than it is high.

In vision, anisotropy in the perception of linear extent reveals itself in the well-known tendency for horizontals to appear smaller than physically equal verticals: the horizontal-vertical illusion, or HVI (see, e.g., Finger \& Spelt, 1947; Higashiyawa, 1992; Künnapas, 1955). Most studies of the HVI have tested horizontal and vertical line segments configured in the shape of an L or a Tconfigurations also commonly used to test haptic length perception. In matching paradigms, for instance, one segment of the $\mathrm{L}$ or the $\mathrm{T}$ is fixed in length, and the subject adjusts the length of the other segment to make the two appear equally long.

The size of the HVI often varies with the configuration (see, e.g., Finger \& Spelt, 1947; Künnapas, 1955), apparently because use of a $T$ configuration conflates horizontal-vertical anisotropy with an illusion produced by bisection. A bisected line segment is perceived to be shorter than a physically equal but uninterrupted line segment (Künnapas, 1955). Indeed, anisotropy is typically greater in $\mathrm{T}$ configurations than in L configurations, in 
haptic perception (Deregowski \& Ellis, 1972) as well as in vision (Cormack \& Cormack, 1974; Day \& Avery, 1970). To avoid (or at least minimize) configural effects of this sort, the present study used individual, isolated, rectilinear stimuli in order to investigate haptic perception of length, focusing on two kinds of anisotropy that have been reported. One is related to the nature of the exploratory motion, and the other is related to the relative spatial position of the arm and hand (or the object explored).

Central to haptic perception is the nature of the exploratory motion (Lederman \& Klatzky, 1987; Lederman et al., 1987). Just as it is possible to specify visual space in terms of horizontal, vertical, and other axes, so is it possible to specify axes of manipulatory space in terms of the haptic motions that are elicited by, or directed toward, various objects. If, for instance, we idealize the body (torso) as cylindrical, motions may be tangential to a circle that is concentric with the torso or radial (perpendicular) to its central axis. Imagine holding your hand directly in front of your body. Moving the hand to the left or the right entails tangential motion, whereas moving it straight ahead, away from the body, entails radial motion.

Much as the HVI in vision refers to an overestimation of verticals, relative to physically equal horizontals, so the radial-tangential effect (RTE) in haptics refers to an overestimation of radial motions, relative to equidistant tangential motions (see, e.g., Cheng, 1968; Davidon \& Cheng, 1964; Day \& Wong, 1971; Marchetti \& Lederman, 1983). In an earlier study, we sought evidence for functional connection between the haptic RTE and the visual HVI (Marks \& Armstrong, 1996). In one experimental condition, we manipulated the size of the haptic RTE by varying the relative extents of radial and tangential movements, and in another condition, we manipulated the size of the visual HVI by varying the relative extents of horizontal and vertical lines. Measuring both the RTE and the HVI in each condition, we found no cross-modal effects; that is, shifts in the size of the RTE had no effect on the HVI, and shifts in the size of the HVI had no effect on the RTE. This outcome is consistent with the hypothesis that the RTE and the HVI derive from independent perceptual mechanisms.

The present study had two goals, the first descriptive and the second explanatory. Experiments 1 and 2 compared radial and tangential motions when both were made in the horizontal plane, quantifying the effect of type of motion on perception of several physical extents. Subjects explored rectilinear stimuli, varying in physical extent, that were oriented either perpendicular to the frontal plane of the body (so subjects would have to make radial motions, toward or away from the body) or parallel to the body (so subjects would have to make tangential motions).

The position or location of an object in space, relative to the body, as well as its orientation, may affect the type of motion exerted (and thus the patterns of muscular activity and the mechanical stresses). When the arm is positioned at different distances from the body, radial mo- tions are overestimated, relative to tangential motions, both when motions are made in front of the body and when they are made on the side of the body (Cheng, 1968) and both when the arm is outstretched and when it is bent (Day \& Wong, 1971). But proximity to, or distance from, the body also counts. When subjects move their hand tangentially through a fixed distance, perceived extent is greater when the hand is near the body than when the hand is farther away. Although Cheng (1968) reported an effect of proximity only with tangential movements, Marchetti and Lederman (1983) found such an effect with radial movements as well. In Experiments 1 and 2, the role of the lateral position of the stimulus was examined, and in Experiment 2, the role of the distance of the stimulus from the body was examined.

It is not yet clear just what matters to proximity. The critical variable could be the degree to which the arm is extended. Or it could be the location of the hand's exploratory movement in space, relative to the body (Bradshaw, Burden, \& Nettleton, 1986; but see Bradshaw, Nettleton, \& Spehr, 1982). Imagine reaching for two objects, equidistant from the body, one located to the left and the other to the right of the midline. If one uses the right arm the arm must extend farther to reach the object on the left than the one on the right, even though the exploratory movements of the hand take place at equal distances from the body. Consequently, if it is extension that counts, proximity should exert an effect, with the object on the right appearing to be greater in extent than the object on the left; but if it is the location of the exploring hand that counts, the perception of extent should be the same in both. In Experiment 2, we sought to dissociate these two senses of proximity by varying both lateral position and distance of the objects being explored.

In this study, we also investigated a matter that, to the best of our knowledge, has not seen investigation heretofore - namely, the stimulus object that elicits the arm and hand movements. Variations in physical dimensions of the object that are independent of length, such as its width or height, may, nevertheless, affect the local tactual and, perhaps, also the kinesthetic inputs. For example, exploring a thin raised line will produce a tactile perception of the line and its background from which it emerges, whereas one does not perceive the background directly when one explores objects with a broader surface, such as solid blocks. Moreover, the position of the wrist, the temporal characteristics of the movements, and perhaps other properties of the exploratory motions may differ and could, thereby, affect perception of linear extent. In Experiment 1, the perception of the extent of lines and that of solid blocks were compared.

Finally, we tested the hypothesis that the RTE depends on temporal differences in exploratory movements and, in particular, on the duration of the movement. Following earlier observations (Lederman et al., 1987; Ono, 1969; Wapner, Weinberg, Glick, \& Rand, 1967), Hollins and Goble (1988) uncovered a direct relation between perceived distance of linear arm motion and duration of 


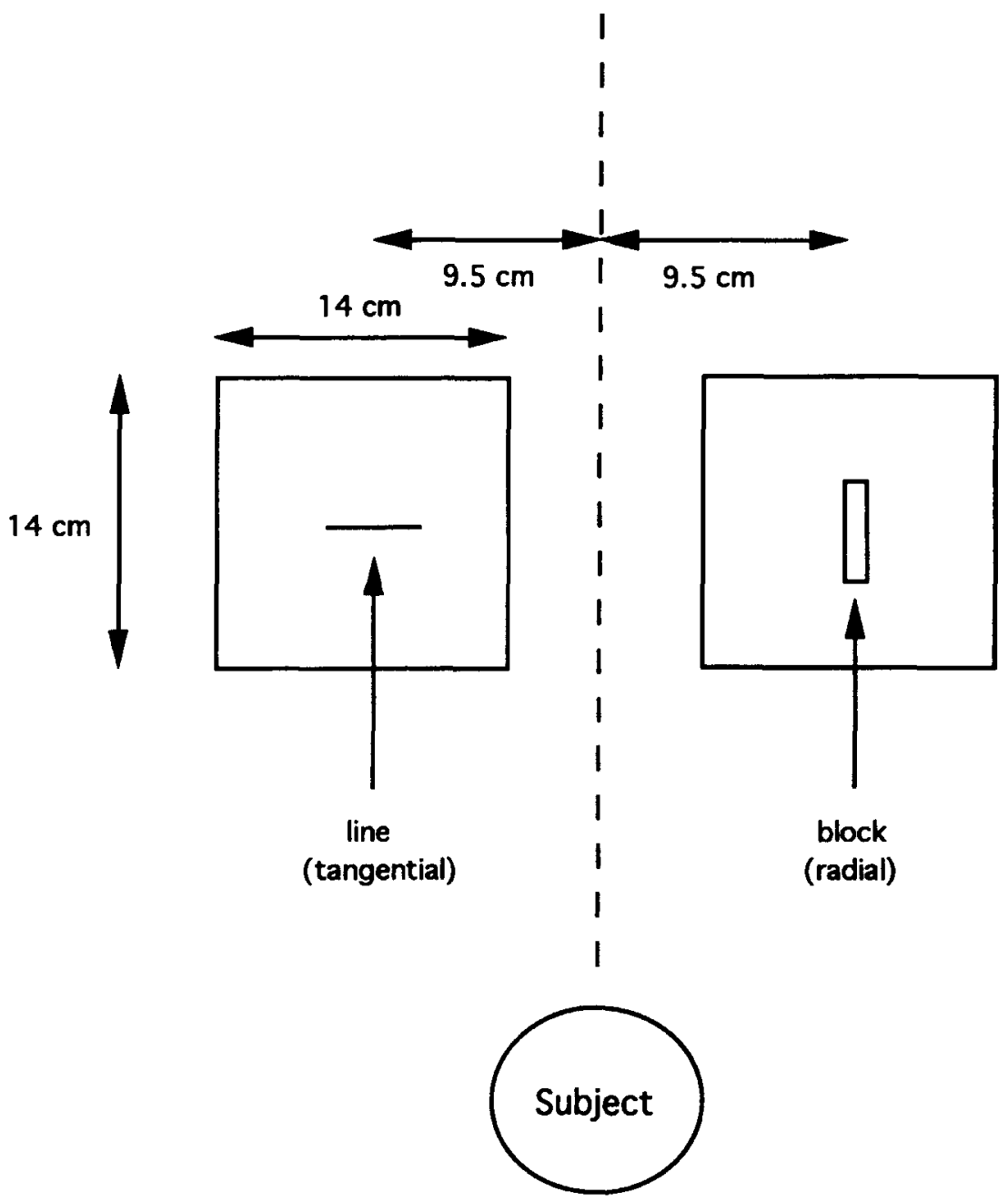

Figure 1. A diagram of the locations of the stimuli in Experiment 1 relative to the subjects. The figure gives examples of a line requiring tangential motion (left) and a block requiring radial motion (right).

movement. Given a fixed linear extent, the greater the time to traverse the extent, the greater the judgment of length. Perhaps subjects execute radial movements more slowly than spatially equivalent tangential movements and, consequently, judge the former to be greater in spatial extent. Experiment 3 tested this hypothesis.

\section{EXPERIMENT 1}

In the first experiment, we evaluated how the type of stimulus object explored (raised line vs. solid block), lateral position (left vs. right of midline), and direction of motion (toward vs. away from the body or the midline) interact with the type of motion (radial vs. tangential) in determining perceived linear extent. To provide a quantitative context to assess these effects, both sets of stimulus objects-lines and blocks-also varied in physical length. Two versions of the experiment were conducted. In Experiment $1 \mathrm{~A}$, the subjects judged the perceived extent of lines and blocks in different test sessions, whereas in Experiment $1 \mathrm{~B}$, the subjects judged all the stimuli within a single session, thereby helping to ensure that all the stimuli were judged on a single, common response scale.

\section{Method}

Subjects. Twenty subjects, paid to participate, were recruited from the Yale community, 6 men and 4 women serving in Experiment $1 \mathrm{~A}$, and another 6 men and 4 women serving in Experiment 1B. The subjects' ages ranged in Experiment $1 \mathrm{~A}$ from 18 to 38 years (mean $=24$ years; median $=23.5$ years) and in Experiment $1 \mathrm{~B}$ from 18 to 32 years $($ mean $=24$ years; median $=$ 24.5 years). All of the subjects in this and the subsequent experiments were right-handed, as determined by unconstrained self-report.

Stimuli. The stimuli consisted of either raised line drawings or solid rectangular blocks constructed of wood, both of which varied in length from 5 to $9 \mathrm{~cm}$ in $1-\mathrm{cm}$ steps. The raised lines were constructed with a Sewell Raised Line Drawing Kit. Drawn at the center of a 14-cm square of transparent plastic, the raised lines were approximately $1 \mathrm{~mm}$ wide, $1 \mathrm{~mm}$ high at the endpoints, and $0.5 \mathrm{~mm}$ high along their extent. The blocks, approximately $10 \mathrm{~mm}$ high and 


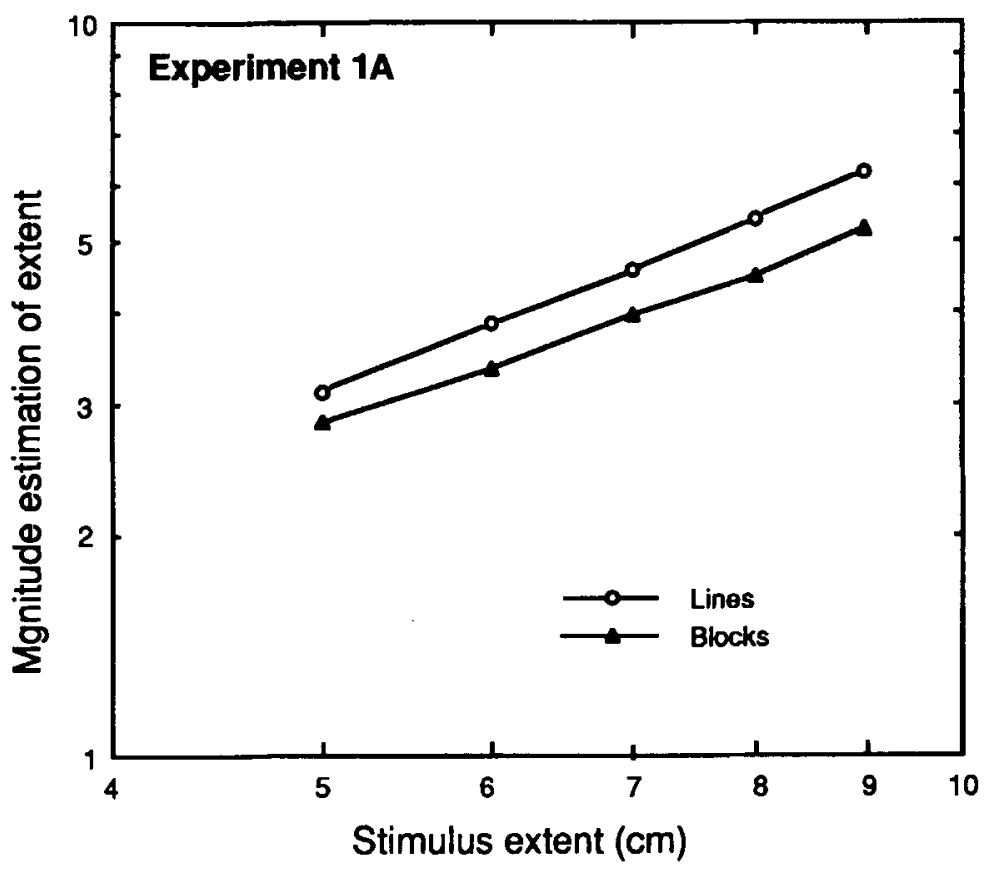

Figure 2. Geometric mean magnitude estimates of perceived extent of raised lines (open circles) and wood blocks (filled triangles), plotted against physical length, in Experiment $1 \mathrm{~A}$. Lines and blocks were presented to the same subjects, but in separate test sessions.

$10 \mathrm{~mm}$ wide, were mounted at the center of $14-\mathrm{cm}$ squares of cardboard. Each stimulus square could be affixed in the horizontal plane to a tray, so that the square's center fell $9.5 \mathrm{~cm}$ to the right or the left of the tray's center. Positions of the stimuli are shown in Figure 1.

Procedure. The subjects sat in front of the tray, which was positioned at the same height as the armrests, so that each subject's forearm was at about elbow level when exploring the stimuli. The subjects were blindfolded and used only their right arms to explore each stimulus, to which they were guided by the experimenter. The subjects explored the stimuli actively but in a proscribed manner, passing over each stimulus once with the index finger of the right hand. In this and all the subsequent experiments, radial motions were toward or away from the body, and tangential motions were left-to-right or right-to-left. After each exploration, the subjects were asked to judge the linear extent by the method of magnitude estimation.

To the first stimulus, the subjects could assign whatever number seemed appropriate to stand for the length, and then, to subsequent stimuli, they were to assign other numbers in proportion, using whole numbers or decimals as needed. The scale was unlimited at the upper end and was limited at the lower end only by zero, which would indicate no perceived length at all. The direction of motion over the stimulus was specified at the start of each trial. Although speed of motion was not strictly controlled, the subjects were encouraged to use a similar speed throughout the experiment. Orientation of the finger was also determined by each subject's preference. Occasionally, the experimenter noticed that the subjects would pause at fixed time intervals during their movements, this perhaps reflecting a strategy that permitted computation of length from the number of pauses; when this happened, the experimenter requested that the subject use a continuous, sweeping motion instead.

In Experiment $1 \mathrm{~A}$, each subject participated in two sessions, held on different days, judging the length of lines in one session and that of blocks in the other, the order of sessions being counterbalanced across subjects. Each set of stimuli numbered 40 , resulting from the factorial combination of each of the five physical extents, presented in two orientations (and, thus, associated with two types of motion, tangential and radial), at two lateral positions (left and right of the subject's midline), and with the subject instructed to use two directions of motion (toward and away from the body with radial motion; toward or away from the body's midline with tangential motion). Within a given session, the entire set of 40 stimuli was given in three replicates, making a total of 120 trials in all. In addition, 10 randomly chosen stimuli served as practice at the start of each session (and practice was so indicated to the subjects). Order of stimuli within each replicate and within the practice set was randomized separately for each subject.

In Experiment 1B, each subject participated in a single session, in which the 80 stimuli ( 40 lines and 40 blocks) were presented in a single replicate and in a different random order for each subject. Introductory practice trials consisted of 6 randomly selected stimuli, 3 lines and 3 blocks.

\section{Results and Discussion}

Experiment 1A. The numerical judgments given by each subject to each stimulus were first pooled logarithmically over replicates. Because analyses indicated that neither lateral position nor direction of motion affected the judgments substantially, the means obtained from each subject were then pooled logarithmically over both position and direction. Figure 2 plots geometric mean magnitude estimates as a function of physical length, showing separately the functions obtained for lines and blocks, pooled over judgments associated with both ra- 


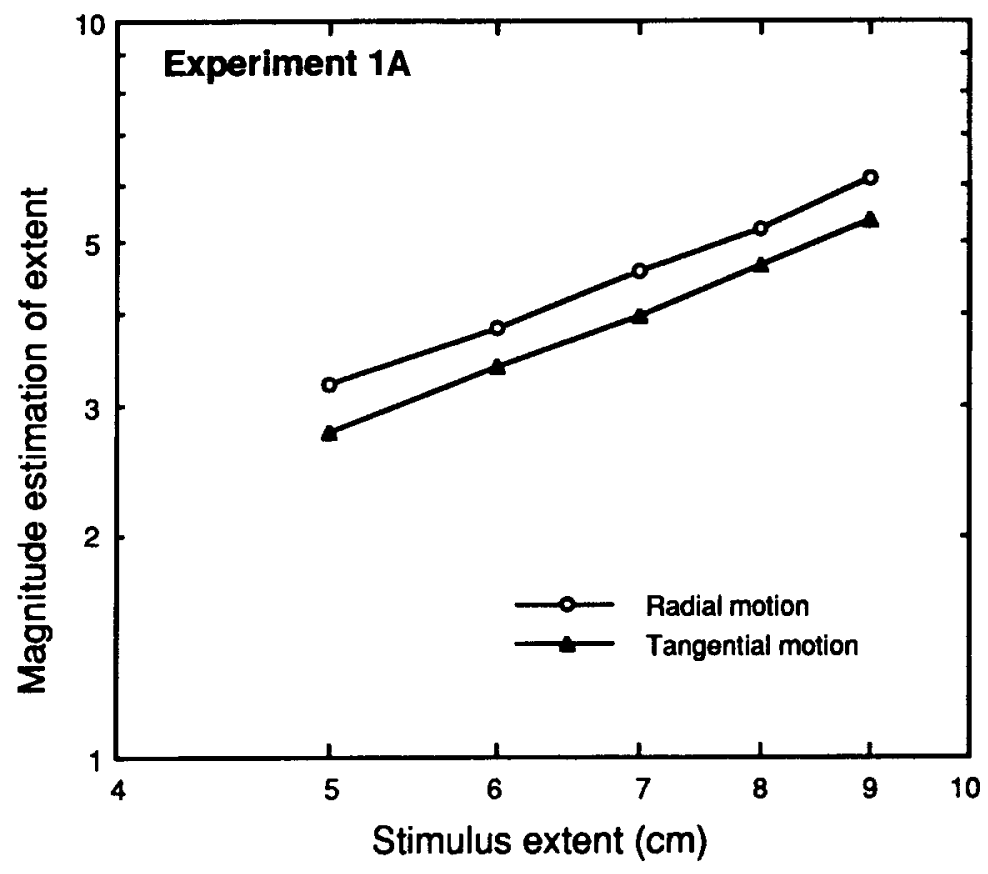

Figure 3. Geometric mean magnitude estimates of perceived extent of radial motions (open circles) and tangential motions (filled triangles), plotted against physical length, in Experiment $1 \mathrm{~A}$. Judgments were pooled over the two test sessions, one of which used lines and the other blocks.

dial and tangential motions. It is clear that, at every length, lines gave greater magnitude estimates, being judged to be, on average, $16 \%$ longer than blocks. By analogy, Figure 3 plots separate functions for radial and tangential motions, in each case pooled over judgments given to both lines and blocks. Stimulus lengths associated with radial motions were judged to be $14 \%$ greater, on average, than lengths associated with equivalent tangential motions (14.6\% for lines and $13.6 \%$ for blocks).

It is important to recognize that percentage differences in magnitude estimates of extent need not be mathematically equivalent to the measure typically reported from perceptual matches - namely, percentage differences in radial and tangential stimulus extents that are judged to be perceptually equal. In principle, the two measures will be equivalent only if the judgments of extent - here, magnitude estimates - are proportional to physical extent. Fortunately, magnitude estimates of linear extent generally follow such a rule, at least approximately, and the present results are no exception. Note that the function's exponent (its $\log -\log$ slope) is slightly greater for lines (1.17) than for blocks (1.01), although more nearly the same for radial movements (1.08) and tangential movements (1.11). Just as important as the slopes are the differences in log intercepts, which in part reflect differences in perceived lengths of lines versus blocks and radial versus tangential motions. Unfortunately, when the slopes vary, the relation between intercepts is not invariant but depends on the choice of stimulus unit - that is, on the stimulus defined to have unit value. Thus the log intercepts (common log- arithms) for radial and tangential motions (functions in Figure 3 ) would equal -0.258 and -0.334 (difference $=$ 0.076 ), respectively, if the stimuli were measured in centimeters; but because the functions have slightly different slopes, the corresponding log intercepts would equal 1.913 and 1.884 (difference $=0.029$ ), were the stimuli measured in meters. Perhaps the functionally most useful assessment of relative intercepts is made when the unit is selected to fall at the center of the log stimulus range tested. Under these conditions, the log intercepts for lines and blocks are 0.643 and 0.581 (difference = 0.062 , corresponding to $15 \%$ ), respectively, and the log intercepts for radial and tangential motions are 0.639 and 0.583 (difference $=0.056$, corresponding to $14 \%$ ).

One interpretation of the proximity effect in haptic perception - that greater perceived extent is associated with tangential motions when the hand is closer to the body-leads to the prediction that stimuli placed to the right of midline, being closer to the right hand doing the exploration, should be judged to be greater than equivalent stimuli placed to the left of midline. But the results did not bear out this prediction. Indeed, the judgments of extent associated with tangential motions were slightly $(5 \%)$ greater when the objects were located on the left, rather than on the right, although this trend was not significant. With radial motions, judgments were virtually identical (as were different directions of motion, away being only $1 \%$ greater than toward, whether radial or tangential). Perhaps what matters in proximity is the position of the stimulus relative to the body. 
The geometric mean magnitude estimates were evaluated statistically by an analysis of variance (ANOVA), calculated on logarithmically transformed values, using the five within-subjects variables: type of stimulus (lines and blocks), extent $(5,6,7,8$, and $9 \mathrm{~cm})$, type of motion (radial and tangential), lateral position (right and left), and direction of motion (toward and away from the torso when the motion was radial, toward or away from the midline when the motion was tangential). This analysis showed three reliable main effects: (1) of stimulus, consistent with greater judgments given to raised lines than to blocks $[F(1,9)=16.33, p<.003] ;(2)$ of extent, perceived length increasing systematically with physical length $[F(4,36)=124.68, p<.001] ;{ }^{1}$ and $(3)$ of motion, reflecting greater lengths associated with radial motions than with tangential motions $[F(1,9)=48.76, p<.001]$. Neither direction $[F(1,9)<1]$ nor position $[F(1,9)=1.74$, $p>.20]$ produced a reliable main effect.

The ANOVA also showed two reliable interactions: (1) between stimulus and extent, consistent with the difference between the $\log -\log$ slopes of the functions in Figure $2[F(4,36)=4.265, p<.05]$ and implying that the percentage difference in the perceived extent of lines and blocks was not constant but instead grew slightly with increasing physical length; and (2) between type of motion and position $[F(1,9)=5.75, p<.05]$, suggesting that the RTE was slightly greater when stimuli were presented on the right, rather than on the left. Post hoc measures of simple effects showed that the effect of extent was reliable for both lines $[F(4,36)=138.88, p<.001]$ and blocks $[F(4,36)=83.22, p<.001]$, that the effect of stimulus was reliable at every length [for increasing lengths, $F(4,36)=$ $5.68,39.85,10.73,18.61$, and 13.97 , all values of $p \leq$ $.05]$, and that the effect of direction was reliable at both the left position $[F(1,9)=31.12, p<.001]$ and the right position $[F(1,9)=39.14, p<.001]$, but that the effect of position only approached significance with tangential motion $[F(1,9)=4.66, p<.06]$ and was nonsignificant with radial motion $[F(1,9)<1]$. Note that the small difference between the RTEs in lines and blocks (interaction of stimulus $\times$ motion) was trivial $[F(1,9)<1]$.

Besides confirming the presence of a clear difference between extents perceived through tangential motions and those perceived through radial motions, Experiment $1 \mathrm{~A}$ revealed the novel, and somewhat fortuitous, difference in the haptic perception of lines and blocks. It is necessary, however, to interpret this difference with caution. Unlike the greater linear extents associated with radial versus tangential motions, which appeared in judgments obtained within each test session, the greater judgments given to raised lines, as compared with blocks, were obtained across sessions. Thus, the difference between judgments of lines and those of blocks might represent nothing more than a fortuitous difference in the mean size of the numbers used by the subjects in the two sessions. Accordingly, in Experiment 1B, we compared perception of lines and blocks within the same test session and examined further the role of lateral position of the stimulus.
Experiment 1B. Figure 4 plots the geometric mean magnitude estimates obtained with lines and with blocks against physical length. As in Experiment $1 \mathrm{~A}$, the lines were judged to be greater in extent than the blocks, although now only $7 \%$ longer. Linear extents associated with radial motions were judged to be $11 \%$ greater than those associated with tangential motions $(10.5 \%$ for lines and $11.4 \%$ for blocks; see Figure 5). The exponents of the functions (log-log slopes) are uniform, equaling 1.08 and 1.11 for lines and blocks (Figure 4) and 1.09 and 1.06 for radial and tangential motions (Figure 5), and the log intercepts, calculated with unit length located at the geometric center of the range, are again greater for lines than for blocks $(0.606$ vs. 0.575 , a difference of 0.031 , corresponding to $7 \%$ ) and greater for radial than for tangential motions ( 0.612 vs. 0.568 , a difference of 0.044 , corresponding to $11 \%$ ).

The data were subjected to an ANOVA, again calculated on logarithmically transformed values, using the five within-subjects variables: stimulus, extent, motion, position, and direction. Now, only three terms, all main effects, were reliable: (1) stimulus $[F(1,9)=7.28, p<$ $.025]$, reflecting the greater perceived length of raised lines; (2) extent $[F(4,36)=83.19, p<.001]$; and (3) motion $[F(1,9)=15.90, p<.0035]$, reflecting the greater judgments associated with radial than with tangential motions. Neither position $[F(1,9)<1]$ nor direction of motion $[F(1,9)=3.59, p>.09]$ had a reliable effect. Unlike Experiment 1A, none of the interactions in Experiment $1 \mathrm{~B}$ was reliable. Thus, the difference between log$\log$ slopes obtained with lines and blocks in Experiment $1 \mathrm{~A}$ (stimulus $X$ extent interaction) was not reliable here $[F(4,36)<1]$ and probably reflected, in Experiment $1 \mathrm{~A}$, the use of different numeric scales in different test sessions. Nor was the small interaction between type of motion and lateral position found in Experiment $1 \mathrm{~A}$ evident here $[F(1,9)<1]$. Lastly, the very small difference between RTEs in lines and blocks (stimulus $X$ motion interaction) was trivial $[F(1,9)<1]$.

The difference between the judged extents of lines and blocks themselves, albeit still substantial, was only about half as large in Experiment 1B as in Experiment 1 A (7\% vs. $16 \%$ ); by contrast, the difference between judgments given to radial motions and those given to tangential motions was comparable in size in the two experiments ( $11 \%$ and $14 \%$, respectively). However, when assessed by an ANOVA performed on the data of both experiments (treating experiment [1 A vs. 1B] as a betweensubjects variable), neither difference varied reliably across experiments-for the experiment $\times$ stimulus interaction $[F(1,18)=2.75, p>.10]$ and for the experiment $\times$ motion interaction $[F(1,18)<1]$. Thus, it is not possible to infer statistically from these results that the difference between lines and blocks is smaller in Experiment 1B than in Experiment $1 \mathrm{~A}$ - although the inability to draw this inference may simply reflect the relatively low statistical power of the between-subjects comparison. More importantly, however, Experiment 1B confirmed two 


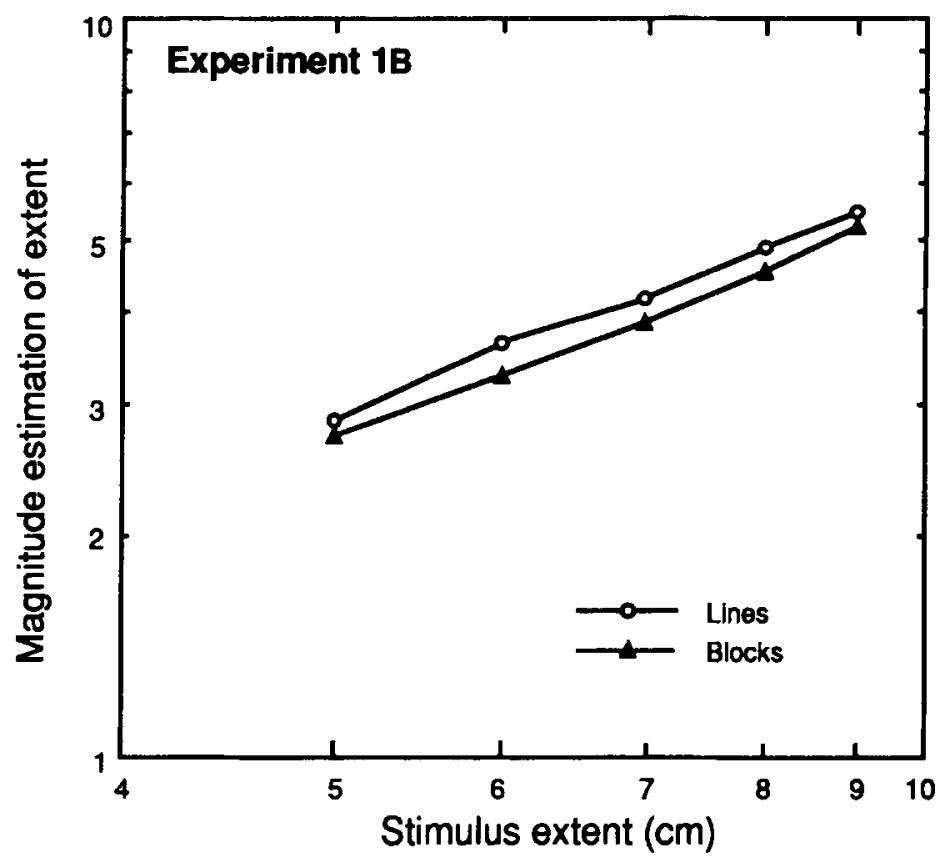

Figure 4. Geometric mean magnitude estimates of perceived extent of raised lines (open circles) and solid blocks (filled triangles), plotted against physical length, in Experiment $1 B$. All the stimuli were presented in a single test session.

main findings of Experiment $1 \mathrm{~A}$, showing how both type of stimulus and type of arm motion influence the perception of linear extent.

\section{EXPERIMENT 2}

Experiment 1 provided little support for the expectation that judgments of stimuli placed to the left and to the right of midline might show the previously reported effect of proximity, greater length being associated with greater proximity. We had anticipated that perceived length might be greater when stimuli were located on the right side, the right hand then being less extended. Perhaps perceived length depends on the proximity of the hand to the body, rather than on degree of arm extension. In earlier studies (e.g., Cheng, 1968), proximity was manipulated by varying distance from the body. Accordingly, Experiment 2 manipulated proximity in two ways-by varying once more the lateral position (more accurately, arm position, defined by locations on an arc concentric with the subject's shoulder) and by varying the distance (from the shoulder). In this manner, it should be possible to distinguish effects of the distance of the stimulus from the body (or, equivalently, extension of the arm) from effects of the position of the object (or, equivalently, location of the hand).

\section{Method}

Subjects. Six men and 10 women, recruited as before, participated. Their ages ranged from 18 to 45 years (mean $=24$ years; median $=20.5$ years). None had served in Experiment 1 .
Procedure. Experiment 2 used the blocks only, the five lengths being the same as those in the earlier experiments. Stimuli took one of nine locations, based on the factorial combination of three positions and three distances. Thus, for each position (left, center, or right), there were three possible locations, corresponding to the different distances (near, middle, and far). For each position, these locations all fell on an imaginary line emanating approximately from the center of the subject's shoulder; consequently, when the subject reached toward a stimulus in a given position (left, right, or center), the direction of the arm movement was roughly constant, independent of the stimulus distance.

The layout of the stimuli is sketched in Figure 6. The three stimuli on the left were positioned approximately on a line from the subject's right shoulder, forming an angle of $30^{\circ}$ from the horizontal, the three stimuli at the center on a line $60^{\circ}$ from the horizontal (which put these stimuli near the midline of the body), and the three stimuli at the right on a line $110^{\circ}$ from the horizontal. These angular positions were fixed and constant for all the subjects. The three distances, however, varied from subject to subject with the length of the subject's arm: The far distance corresponded to each subject's arm when fully extended; the near distance fell on the front of the table and was fixed for all the subjects approximately $20 \mathrm{~cm}$ from the torso; and the middle distance was determined for each subject to fall halfway between the near and the far distances. The distance between the near and the far stimuli ranged from 18 to $38.5 \mathrm{~cm}$ (mean $=27.0$, median $=28)$ at the left, from 22.5 to $45 \mathrm{~cm}$ (mean = 33.9 , median $=34.3$ ) at the center, and from 22.5 to $46 \mathrm{~cm}$ (mean $=$ 33.1 , median $=32.5$ ) at the right. Once more, there were two stimulus orientations (requiring radial or tangential motions). Figure 6 shows, at each location, an example of one stimulus orientation or the other. Hence, a factorial combination of stimulus values yielded 90 stimuli in all, presented in two randomly ordered replicates, for a total of 180 trials. Again, practice trials, now 3 in number, introduced each session and were not counted. In Experiment 1, we observed that some subjects tended to "overshoot" beyond the end of the stimulus. To compensate for this tendency, the subjects in Ex- 


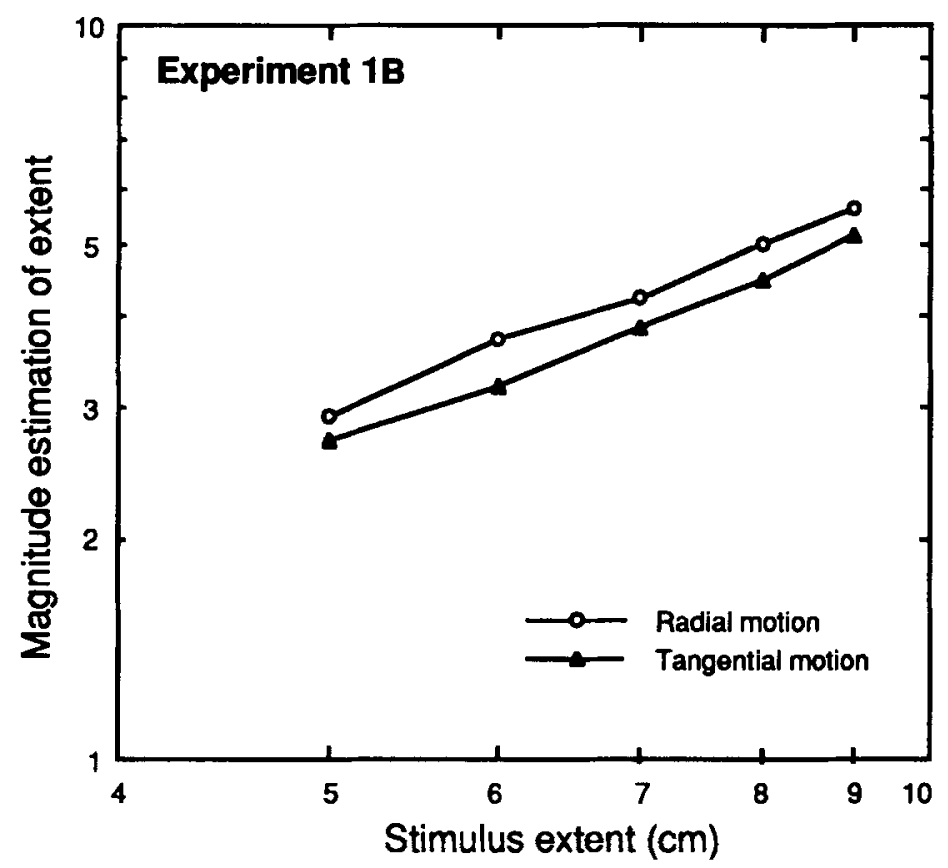

Figure 5. Geometric mean magnitude estimates of perceived extent of radial motions (open circles) and tangential motions (filled triangles), plotted against physical length, in Experiment $1 B$.

periment 2 could pass back and forth over each stimulus, as often as they desired, with the tip of the right index finger. Otherwise, the procedure followed that of Experiment 1.

\section{Results and Discussion}

Figure 7 plots the geometric mean magnitude estimates against physical length associated with radial and tangential motions, respectively. Once again, the results reveal an RTE, with the judgments of radial motions on average being $7.1 \%$ greater than the corresponding judgments of tangential motions. On the whole, the overall distance of the object, and thus the distance of arm system from the body, mattered little, if at all, although the position of the arm did. Radial motions were perceived as being $7 \%$ longer on the left than on the right. In contrast, tangential motions were perceived as being $4 \%$ greater on the right than on the left.

Judgments were pooled over replicates, and an ANOVA was performed on the logarithmically transformed values, using four within-subjects factors: motion (radial, tangential), position (left, center, right), distance (near, middle, far), and extent $(5,6,7,8$, and $9 \mathrm{~cm})$. Two main effects were significant: (1) type of motion $[F(1,15)=39.49, p<$ $.001]$, radial motions being rated as being longer than tangential motions, and $(2)$ extent $[F(4,60)=147.68, p<$ $.001]$. Neither the main effect of position $[F(1,15)<1]$ nor that of distance $[F(1,15)<1]$ was significant. The motion $\times$ position interaction was significant $[F(2,30)=$ $12.91, p<.0015]$. Post hoc measures of simple effects indicate that position had a significant effect with radial motions $[F(2,30)=9.44, p=.001]$, judgments being greater on the left than on the right, but only a marginal effect with tangential motions $[F(2,30)=3.015, p<.065]$, judgments being greater on the right than on the left. Lastly, the RTE was substantial and reliable in the left and central positions $[F(1,15)=36.73$ and 26.33 , respectively, both values of $p<.001]$, but smaller and not quite reliable in the right position $[F(1,15)=3.61, p<.08]$.

Why the RTE should be greater on the left in this experiment but greater on the right in Experiment $1 \mathrm{~A}$ is a puzzle. A reviewer suggested the possibility that the difference might stem from the different proportions of women and men in the subject populations of the two experiments, relatively more women participating in Experiment 2 . However, women and men performed similarly in both experiments: Both women and men in Experiment 1 A showed larger RTEs with blocks in the right versus the left position, and both women and men in Experiment 2 gave RTEs that were greatest at the left position and smallest at the right. Thus, gender cannot account for the difference between the results of the two experiments.

\section{EXPERIMENT 3}

Perhaps the crucial variable modulating haptic perceived extent is the time taken to examine each stimulus. For example, it was our impression that the subjects in Experiment 1 found it more difficult to follow the raised lines than the solid blocks. On occasion, the subjects unwittingly strayed from the lines, successful exploration of which requires considerable effort and caution. It is conceivable that the relatively rough texture of the 


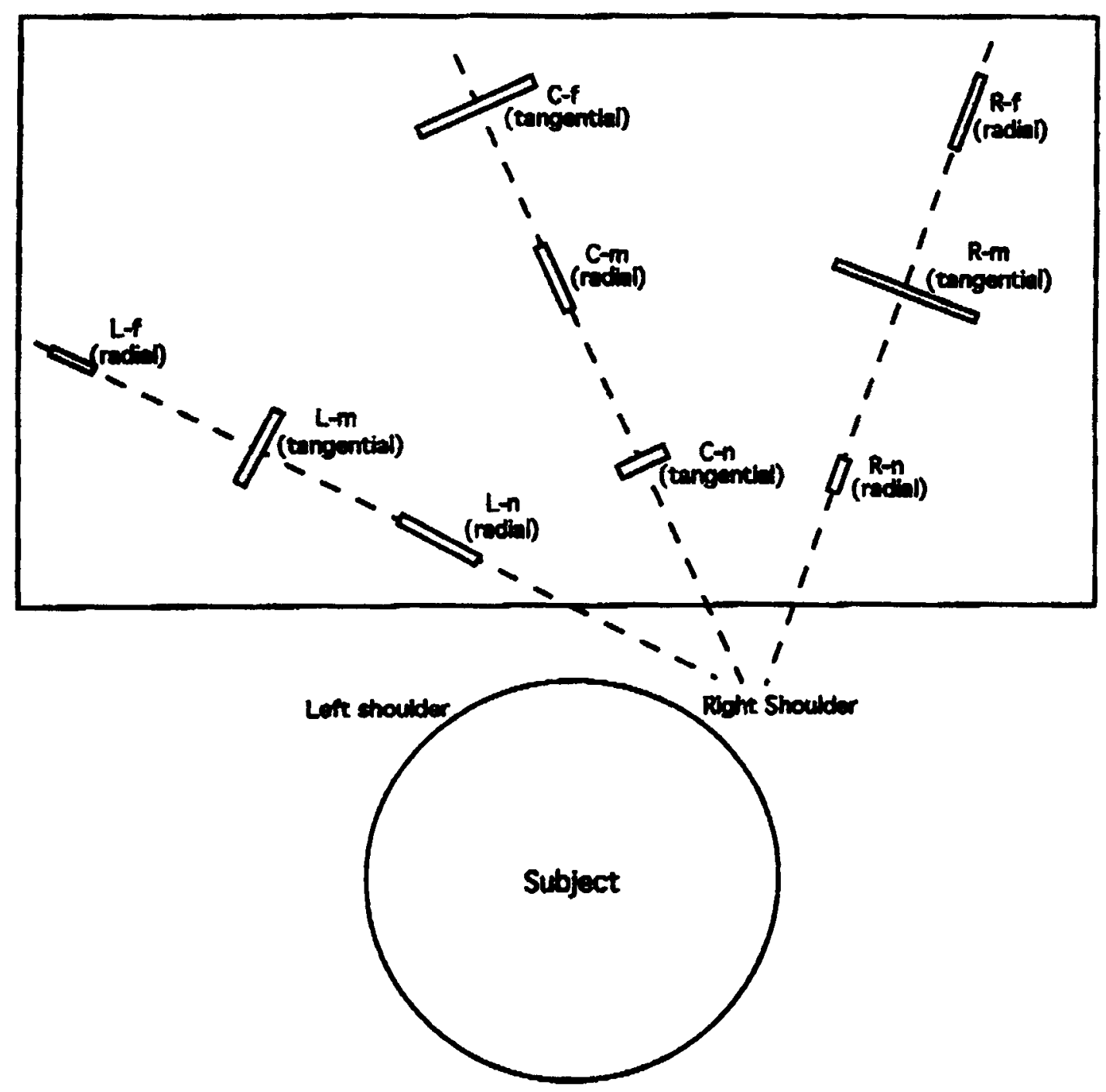

Figure 6. A schematic diagram of the locations of the stimuli in Experiment 2. Stimuli could be placed at three positions, on the left (L), in the center, (C), or on the right (R), and at three distances, near (n), middle (m), or far (f). The figure gives examples of stimuli requiring either a radial or a tangential motion at each of the nine possible locations.

lines, as compared with the smooth blocks, contributed to such differences. The subjects appeared to explore the rigid blocks with ease and, often, rapidly. Indeed, rather than pausing when they reached the end of the stimulus, excessive speed often led the subjects to sweep their hands beyond the end of the blocks.

Several studies indicate that subjects judge extents to be greater when active movements are slower (Hollins \& Goble, 1988; Ono, 1969; Wapner et al., 1967; Wong, 1977), a phenomenon reminiscent of the tau effect described by Helson and King (1931). Furthermore, the RTE has been linked to different time cues associated with the two motions (Lederman et al., 1987; Reid, 1954; Wong, 1977). Perhaps both of the main perceptual differences observed in our experiments - the difference between lines and blocks and the difference between radial and tangential motions - are based on temporal cues. It is a rel- atively straightforward matter to investigate the role of temporal cues in the RTE. Consequently, in Experiment 3, we tested the hypothesis that the RTE is directly related to time for exploratory motion. To this end, we used a variant of the method of Hollins and Goble, in which subjects judge perceived extents when instructed to move their arms at different speeds through various fixed distances.

\section{Method}

Subjects. Thirteen women and 11 men served as subjects. Their ages ranged from 19 to 54 years $($ mean $=29$ years; median $=$ 27 years). None had served in Experiments 1 and 2.

Procedure. For Experiment 3, we constructed a device like that used by Hollins and Goble (1988). We mounted two stops, one fixed and the other variable, onto a smooth, lubricated optical bench, on which also rested a freely sliding wedge, which the subject could grasp and move between the stops. Varying the location of the adjustable stop made it possible to vary the distance from 


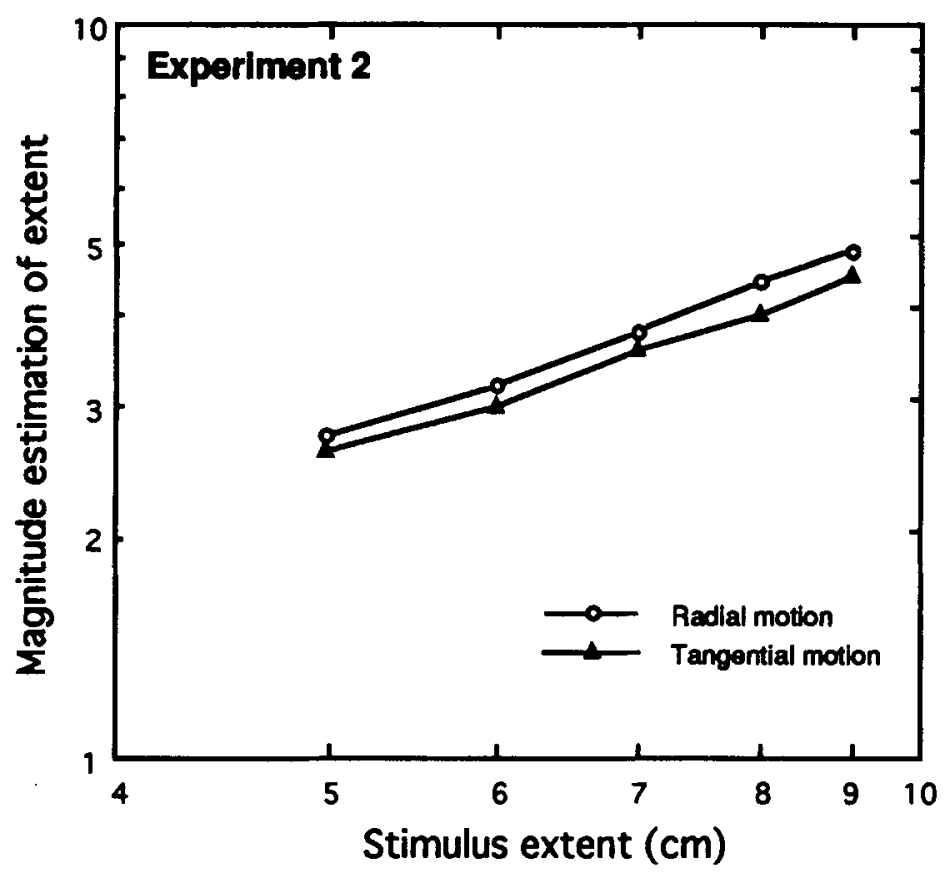

Figure 7. Geometric mean magnitude estimates of perceived extent of radial motions (open circles) and tangential motions (filled triangles), plotted against physical length, in Experiment 2.

the fixed stop. Timing was accomplished by sending a $1000-\mathrm{Hz}$ signal to a frequency counter through microswitches attached to each of the stops; timing began when the wedge was moved from the fixed stop and ended when the wedge reached the adjustable stop.

On each trial, the orientation of the apparatus was set so as to permit radial or tangential motion, the orientation being chosen ran- domly from trial to trial. Each movement started from the same fixed location, directly in front of the blindfolded subject, with direction of motion being either away from the subject (radial) or leftto-right (tangential). There were eight possible lengths traversed$3,7,11,15,19,23,27$, and $31 \mathrm{~cm}$. On half of the trials, the subjects were instructed to use a fast movement, and on the other half of the trials, to use a slow movement. Before the first trial, the experi-

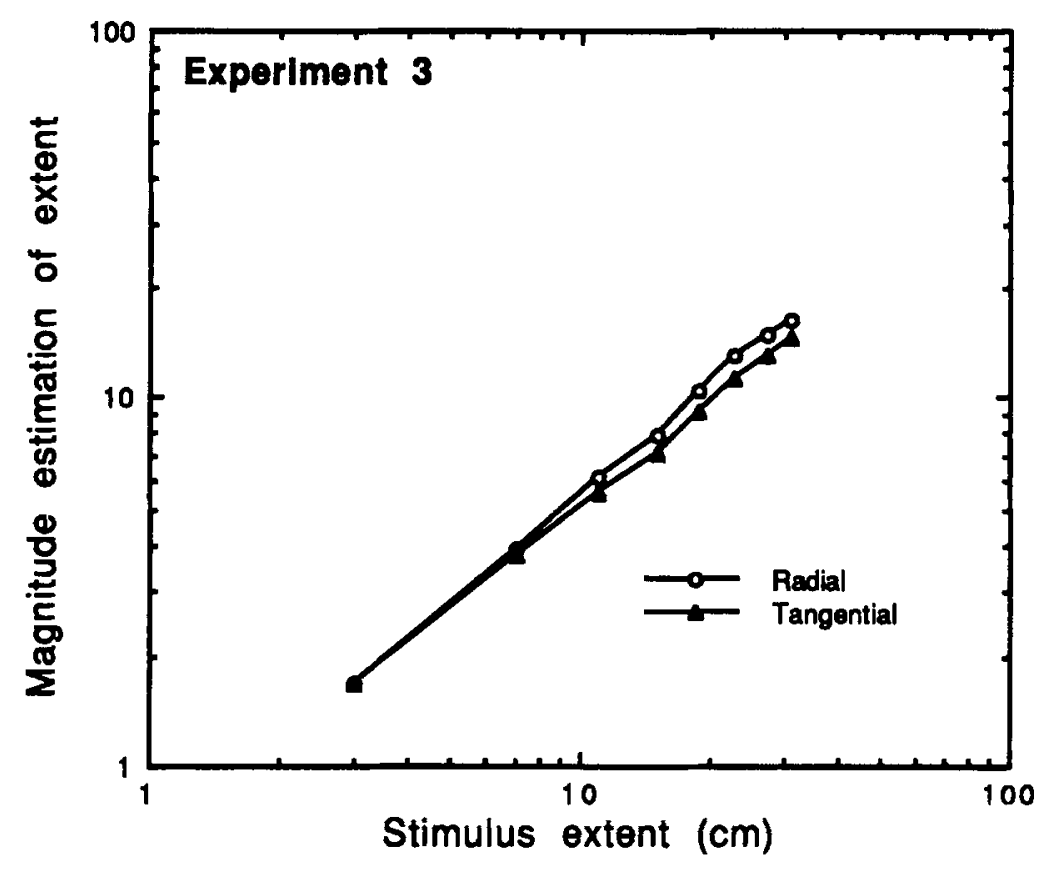

Figure 8. Geometric mean magnitude estimates of perceived extent of radial motions (open circles) and tangential motions (filled triangles), collapsed over speed of movement, plotted against physical extent, in Experiment 3. 


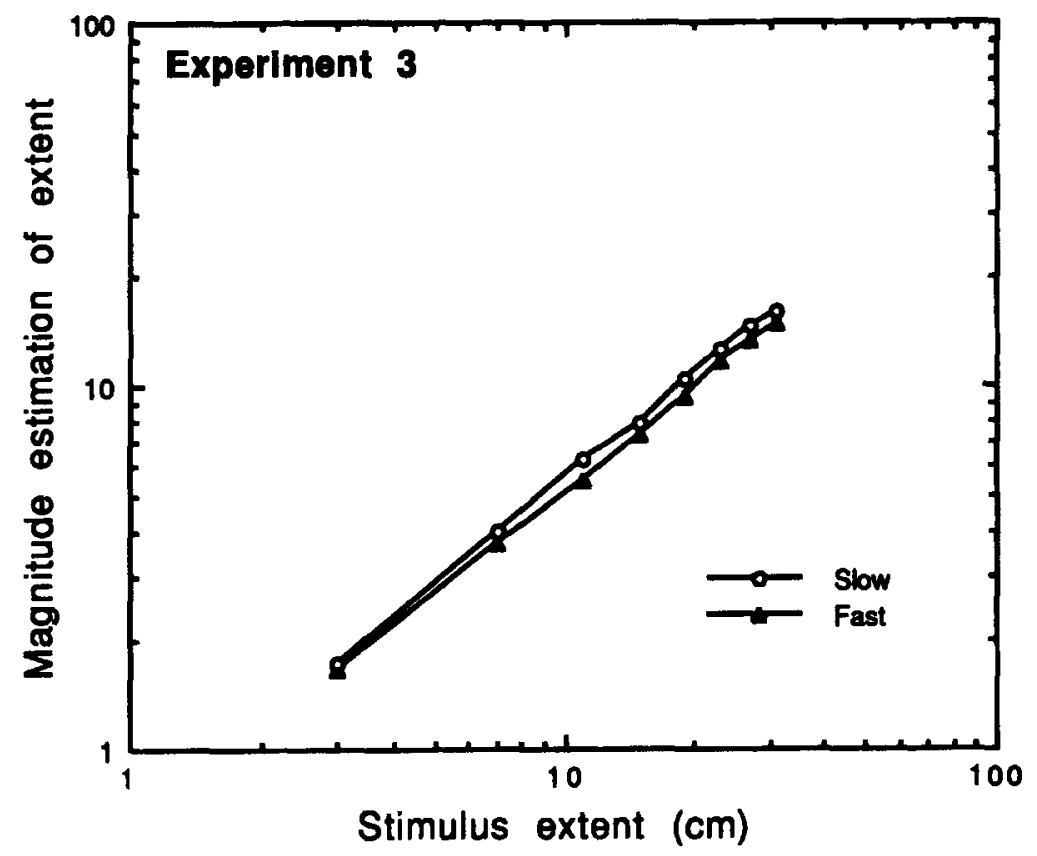

Figure 9. Geometric mean magnitude estimates of perceived extent of slow movements (open circles) and fast movements (filled triangles), collapsed over radial and tangential motions, plotted against physical extent, in Experiment 3.

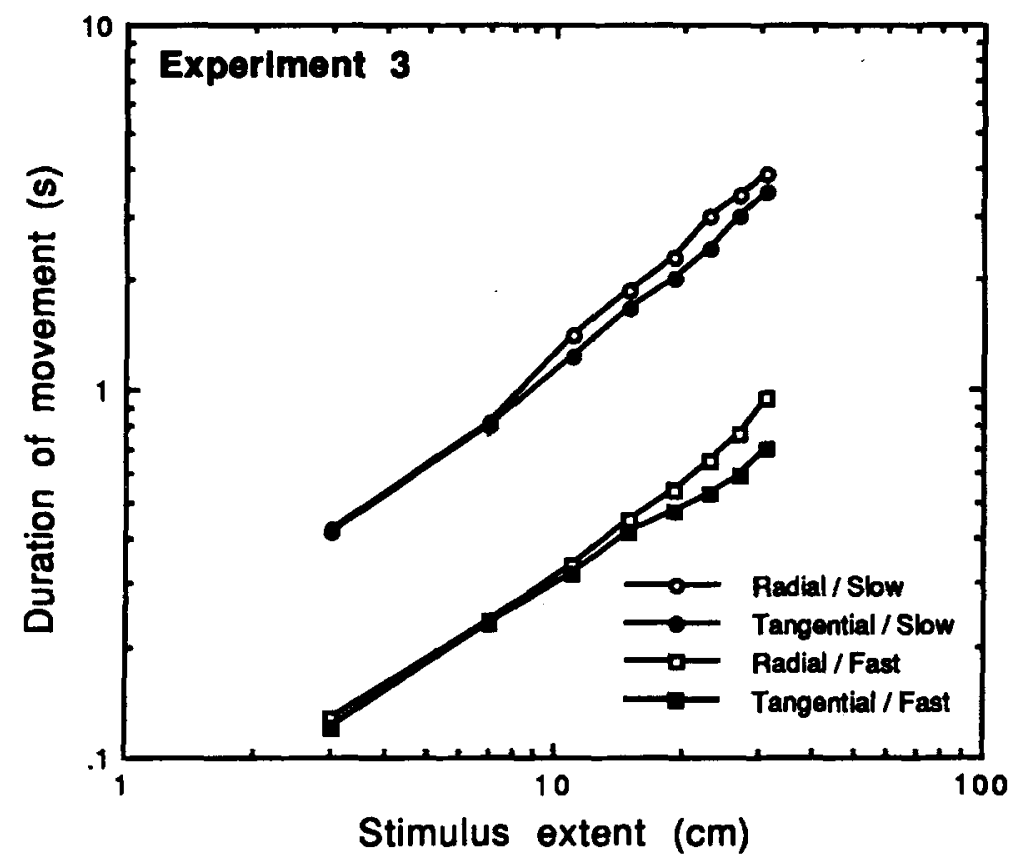

Figure 10. Geometric mean durations of movements with radial motions (open symbols) and tangential motions (filled symbols), made under instructions for the movements to be slow (circles) or fast (rectangles), plotted against physical extent, in Experiment 3. 


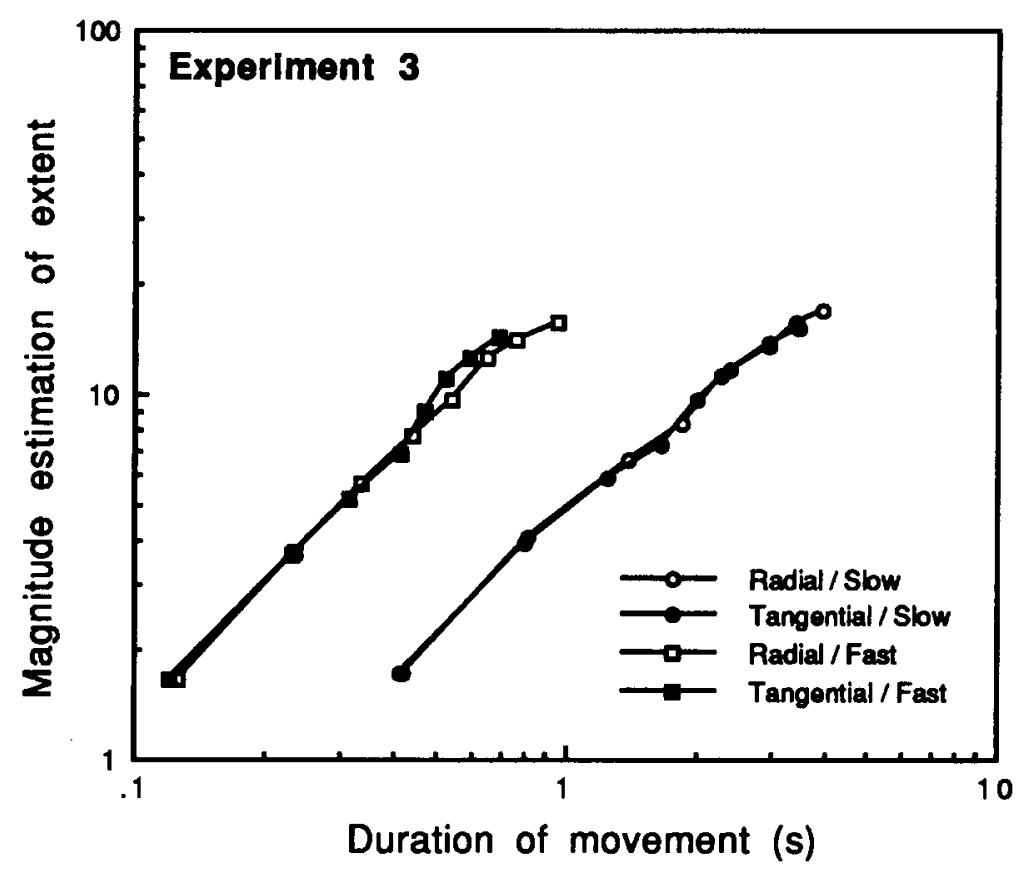

Figure 11. Mean magnitude estimates of extent plotted against mean durations of movement, separately for radial motions (open symbols) and tangential motions (filled symbols) made under instructions for the movements to be slow (circles) or fast (rectangles), in Experiment 3.

menter gave examples of fast (about $30 \mathrm{~cm} / \mathrm{sec}$ ) and slow (about $7.5 \mathrm{~cm} / \mathrm{sec}$ ) by moving the wedge while the subject held it.

Each experimental session contained three replicates of 32 randomly ordered trials (eight lengths $\times$ two orientations $\times$ two speeds). The subjects were permitted only a single movement on each trial, the duration of which was monitored and recorded. As before, the subjects gave numerical estimates of the length traversed on each trial.

\section{Results and Discussion}

The geometric mean estimates of length are plotted against the physical extent-in Figure 8, separately for radial and tangential motions (collapsed over speed of movement), and in Figure 9, separately for fast and slow speeds of movement (collapsed over radial and tangential motions). Both effects are modest in size, amounting, on average, to $9.9 \%$ for orientation and $9.3 \%$ for speed, and both tending to be greater when the physical extents are greater. Note that the size of the RTE in this experiment falls within the range of values found in Experiments 1A, $1 \mathrm{~B}$, and 2 (where it equaled $14 \%, 11 \%$, and $7 \%$, respectively), despite the differences in apparatus and procedure. In Experiments 1 and 2, the subjects judged the linear extent of objects that they explored, whereas in Experiment 3, the subjects judged the length (or distance) traversed between two points. It seems likely that the factors responsible for the RTE are similar, probably the same, across these experimental conditions.

That the critical factor may be temporal is suggested by Figure 10, which plots the geometric mean durations of motion against stimulus extents for each of the combi- nations of orientation and overall (instructed) speed. At both speeds, but especially at the fast one, the subjects did, in fact, execute radial motions more slowly (durations $13.3 \%$ greater) than they did spatially equivalent tangential motions $(11.8 \%$ greater at the slow speed and $15.7 \%$ greater at the fast speed). Finally, Figure 11 plots the magnitude estimates of length against the corresponding durations of motion for each combination of orientation and speed. Note that plotting perceived length against time essentially collapses the functions for radial and tangential orientations at each instructed speed (except, perhaps, for the largest motions at the fast speed). By implication, the differences observed in the time needed to execute movements in different orientations seem largely, if not wholly, to account for the RTE.

We subjected the logarithmically transformed data to a series of ANOVAs. In an initial ANOVA, we examined the judgments of length, using the variables of direction of motion (radial and tangential), speed (fast and slow), and extent (eight values). The results showed all three main effects to be reliable: for motion $[F(1,23)=13.38, p<$ $.0015]$; for speed $[F(1,23)=5.01, p=.035]$; and for extent $[F(7,161)=357.87, p<.001]$. In addition, there was one reliable interaction, of motion $\times$ extent $[F(7,161)=$ $3.86, p<.015]$, reflecting the tendency, apparent in Figure 8, for the RTE to increase as length increases. For all the other interaction terms, $F<1$. Post hoc measures of simple effects showed that the effect of extent was reliable with both radial and tangential motions $[F(7,171)=$ 239.91 and 366.87 , both values of $p<.001]$, whereas the 


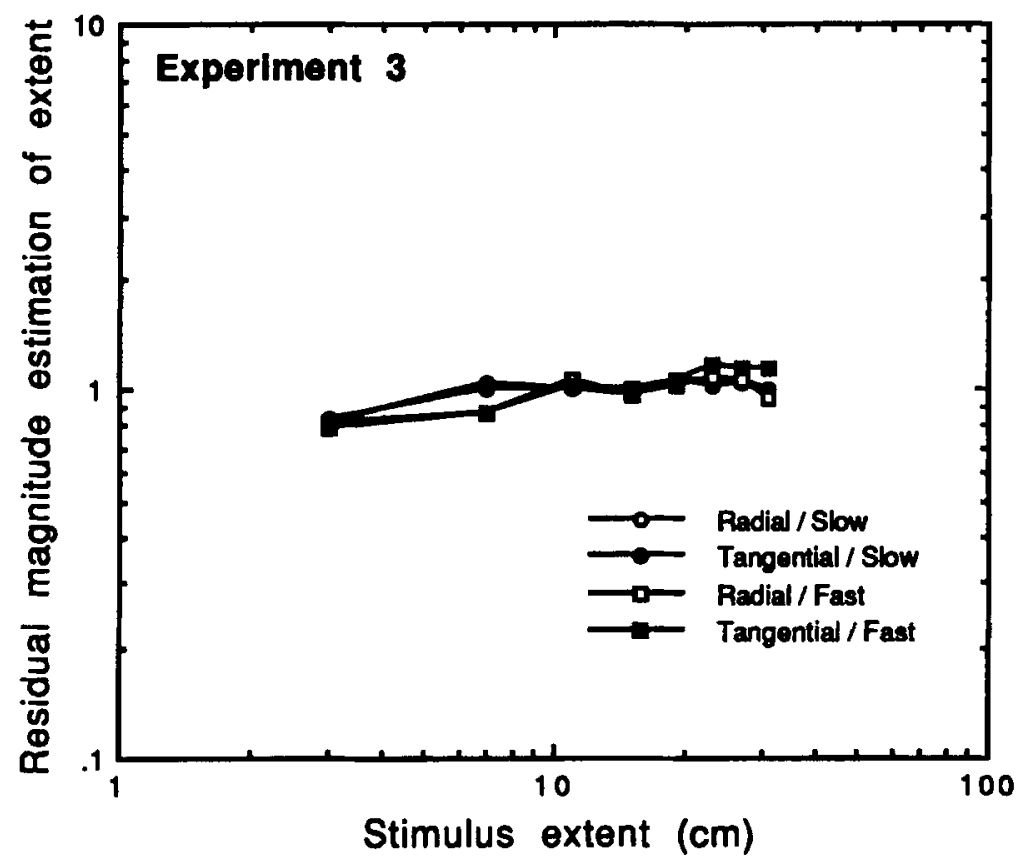

Figure 12. Residuals to the judgments of perceived extent, after removing duration of movement as a covariate, plotted against physical extent, for radial motions (open symbols) and tangential motions (filled symbols) made under instructions for the movements to be slow (circles) or fast (rectangles), in Experiment 3.

effect of motion was nonsignificant at the two smallest extents $[F(1,23)=1.90, p>.18$, and $F(1,23)<1]$ but was reliable at the greater extents $[F(1,23)=6.30,12.13$, $8.27,58.97,16.33$, and 6.34 , all values of $p \leq .02]$.

An analogous ANOVA was applied to the measures of duration, using the same variables, and it too yielded three reliable main effects: for motion $[F(1,23)=16.92, p<$ .001 ; for speed, $F(1,23)=144.38, p<.001$; and for extent, $F(7,161)=510.15, p<.001]$. In addition, there was a reliable speed $\times$ extent interaction $[F(7,161)=6.09$, $p<.001]$, reflecting the tendency, clear in Figure 10, for the $\log -\log$ slope of the functions relating duration to physical extent to be greater with slow than with fast motions. Post hoc measures of simple effects showed the effect of length to be reliable at both slow and fast speeds $[F(7,161)=389.47$ and 231.58 , both values of $p<.001]$ and the effect of speed to be reliable at all eight lengths $[F(1,23) \geq 91.26$, all values of $p<.001]$. The motion $\times$ extent interaction was not quite significant $[F(7,161)=$ $2.08, p=.07]$, and for the remaining two interaction terms, $F<1$.

Next, we sought to assess the influence of duration of movement on the radial-tangential effect by removing the effect of duration as a covariate. To do this, for each subject and separately for each instructed speed, fast and slow, we regressed the log judgments of extent against $\log$ durations of movement of both radials and tangentials. To the extent that duration accounts for the RTE, at each speed a single regression line should account for the relation between duration and length, regardless of orientation. We then used the resulting regression equations in order to remove the predicted (covariate) effects of duration. Note that doing this separately for fast and slow instructed speeds eliminates the main effect of speed (a matter for consideration later). The statistical question was whether the judgments, once transformed in this way, continue to show a residual effect of motion. The results of this final ANOVA, conducted on the transformed data, revealed only a single reliable termnamely, a residual effect of extent $[F(7,160)=6.70, p<$ $.001]$; for all the other terms, $F<1$. Most important, by removing duration as a covariate, we thereby abolished the effect of motion.

Figure 12 plots the transformed data against physical extent. Once the effect of duration was removed, the residual effect of physical extent was small and nonmonotonic, with the judgments reaching a broad peak over the region $11-27 \mathrm{~cm}$. This pattern might reflect variations in the velocities of motion; a residual effect of extent could appear, for example, if the relation between log duration of motion and log distance moved is not strictly linear. Inspection of Figure 10 does suggest some upward curvature in the functions relating duration to extent, especially at the fast speeds and large extents, a result that is roughly consistent with the residual effect. Perhaps, with the longer extents, the subjects slowed their arm movements in anticipation of reaching the end of the stimulus.

The results of Experiment 3 strongly implicate temporal cues in the RTE. Indeed, it is tempting to infer from 


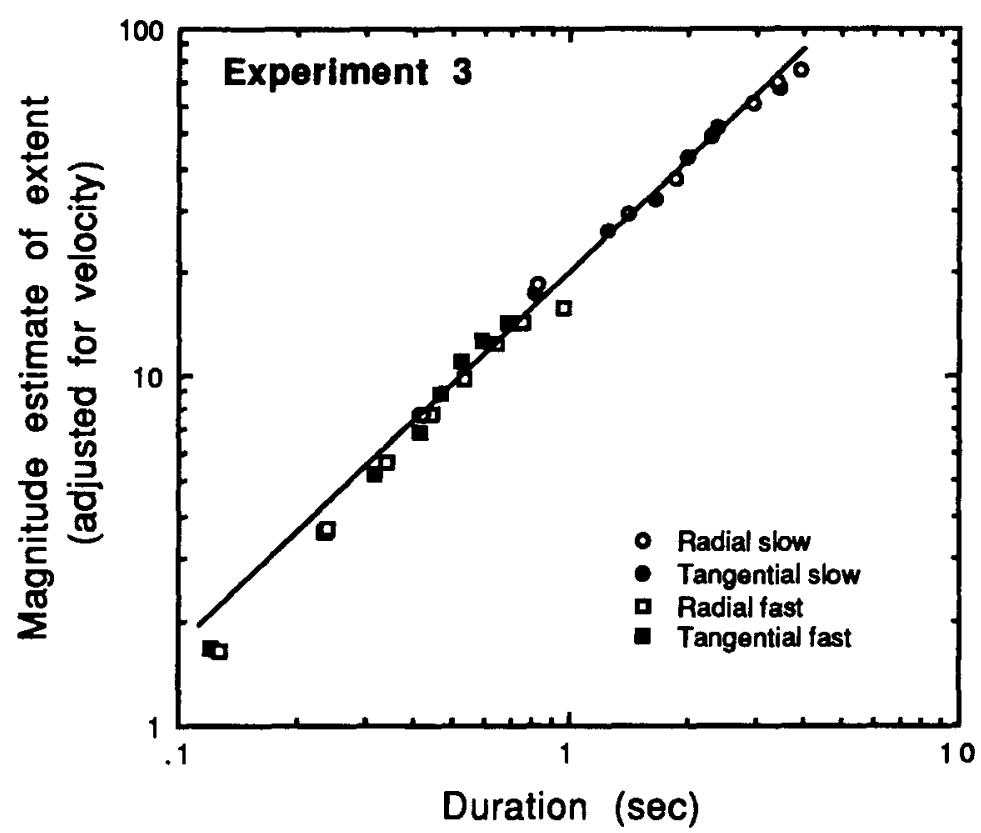

Figure 13. Magnitude estimates of extent plotted against durations of movement, as in Figure 11, but with the judgments obtained with the slow movements increased by a factor of 4.5 , in Experiment 3. The underlying model assumes that subjects base their judgments of extent on the duration of movement, but discount differences in speed.

these results that the difference in perceived extent of radial and tangential arm movements is wholly attributable to the difference in duration of motions in the two orientations. Of course, it is not possible to predict perceived length simply from duration of motion per se. When instructed to move at a fast rather than at a slow speed, the subjects decreased durations by about a factor of four, but this fourfold ratio of durations had only a modest effect on perceived length, as Figure 11 shows. Clearly, the subjects were able to discount, to a great extent, the large difference in their voluntary speed of movement, an ability without which the haptic system might otherwise be called hapless. Figure 13 shows the result of a simple model, albeit presumably one of several that are possible. This model assumes that the judgments of length depend on two factors-namely, duration of movement and speed of movement. In the present model, we assumed that the subjects discounted the effects of slow speeds, relative to fast speeds, by functionally reducing their estimates of extent by a factor of 4.5 (this value was determined by an iterative least-squares procedure). Thus, in Figure 13, the data points for the slow speeds have been increased by this factor $(0.65 \log$ unit), relative to data points for the fast speeds. The straight line represents the equation

$$
\log J=1.106 \log D-0.65 S+1.284
$$

where $J$ is judged extent, $D$ is the duration of the movement, and $S=0$ when the speed is fast and $S=1$ when the speed is slow. As an empirical, first-order account of the data, this simple model, which accounts for $99.3 \%$ of the variance, appears to be adequate.

\section{GENERAL DISCUSSION}

The existence of the RTE has suggested that the perception of haptic space, like that of visual space, is anisotropic: Constant physical distances in different directions or along different axes do not necessarily mark off constant psychological distances. Although the present studies confirm the ubiquitous presence of an RTE in haptic perception, they further suggest that the effect of direction of movement on perceived extent may depend largely, or even wholly, on the dynamics of exploratory motion and, in particular, on the speed with which the movement is executed. Thus, haptic perceptual space may not be intrinsically anisotropic.

Studies by Cheng (1983) and by Marchetti and Lederman (1983) revealed anisotropies based on distance from the subject, as well as on orientation. Cheng investigated the effects of proximity on haptic perception of extent by varying both distance and angular separation, testing both radial and tangential motions. Although Cheng's subjects judged radial motions to be the same across all conditions, tangential motions depended on proximity, with more distant motions judged to be smaller than nearer ones. Marchetti and Lederman later confirmed the effect of proximity with tangential motions, but found that the nearer of two radial motions was judged to be greater as well. 
In Experiment 1, we asked whether such effects of proximity characterize stimuli separated laterally. Given two objects located at equal distances from the body on the left and the right, the right arm must extend farther when it reaches for the object on the left than for the one on the right. If arm extension underlies the effect of proximity, objects on the right should produce greater judgments of extent. Contrary to our expectation, the extents on the left appeared to be as great as those on the right. It is possible, of course, that the absence of a reliable proximity effect here stems from the relatively small magnitude of the lateral separation $(19 \mathrm{~cm})$. In Experiment 2, we found that distance had virtually no effect, but arm position did. Extents of radial motions in the leftmost position were judged to be significantly greater than extents of radial motions in the rightmost position.

In general, the present experiments failed to uncover effects of proximity per se, although the reasons for this remain obscure. We cannot chalk up the absence of such effects to any putative insensitivity in the magnitude estimation method, as it served well to elucidate differences in perception associated with radial and tangential arm movements and, indeed, to reveal differences, not previously known, associated with perception of raised lines and solid blocks.

Both when they were presented independently on separate days and when they were interspersed within the same test session, the subjects judged-and presumably perceived-raised lines to be longer than solid blocks of identical physical length. Why should this be so? Several perceptual features of the two kinds of stimulus could play a role. Because the raised lines are relatively narrow, shallow, and have a "bumpy" texture, the subject's finger contacts both the line itself and much of the surround lateral to it, producing a clear perception of raised figure and background. By way of comparison, the solid blocks are both wider and higher, and, consequently, the subject's finger meets only their surfaces, producing a foreground with no locally sensed background. When examining the blocks, subjects may also elevate the wrist or the elbow relative to its position with the raised lines, perhaps in anticipation of its drop when the finger reaches the end of the block. Finally, the ends of the raised lines were marked with elevated points, which could provide tactile landmarks. We point out, however, if it is not already obvious, that none of these accounts currently has a strong theoretical basis.

Perhaps the simplest explanation, already suggested, would attribute these differences to temporal cues and, in particular, to a tendency for the subjects to take longer to traverse the raised lines and, hence, to judge them as being greater in extent. This explanation would have the virtue of parsimony, as the results of Experiment 3 suggest that the difference in perceived length of radial and tangential excursions may be chalked up largely, if not wholly, to differences in the duration of these movements. In this regard, it is worth noting that the RTE was smallest in Experiment 2 , where the subjects could explore the stimuli repeatedly, a procedure that conceivably could have obscured temporal differences between radial and tangential movements.

Differences in duration of movement were sufficiently large to account substantially for the RTE in Experiment 3, and we suspect that temporal dynamics will account largely or wholly for the effect under other conditions in which it may appear. In this regard, it is conceivable that the RTE itself may characterize some haptic dynamic systems but not others. For example, shoulder-based movement, like those of the present study, may be more subject to illusory effects than are wrist-based movements (Paillard, 1991; see, also, Martinez, 1971). Indeed, it seems likely that the RTE would be small or lacking with movements based on wrist motion, if only because the range of durations would probably be relatively small, and the present results imply that differences in temporal dynamics underlie the effect. Still unknown is the basis for differences in time to execute radial and tangential movements. One possibility is that the amount of time taken to execute movements in different directions reflects biomechanical constraints. Another possibility is that radial and tangential movements are actually perceived differently with respect to duration. In discounting the speed of movement, perhaps the speed of radial movements is overestimated with respect to tangential movements, the result being that distances are overestimated too-although this would leave unexplained the basis for the differences in estimated speed.

\section{REFERENCES}

Bradshaw, J. L., Burden, V., \& Nettleton, N. C. (1986). Dichotic and dichaptic techniques. Neuropsychologia, 24, 79-90.

Bradshaw, J. L., NetTLETON, N. C., \& Spehr, K. (1982). Braille reading in the left and right hemispace. Neuropsychologia, 20, 493-500.

Cheng, M. F. H. (1968). Tactile-kinesthetic perception of length. American Journal of Psychology, 81, 74-82.

CORMACK, E. O., \& CoRmaCK, R. H. (1974). Stimulus configuration and line orientation in the horizontal-vertica! illusion. Perception \& Psychophysics, 16, 208-212.

DAVIDON, R. S., \& CHENG, M. F. H. (1964). Apparent distance in a horizontal plane with tactile-kinesthetic stimuli. Quarterly Journal of Experimental Psychology, 16, 277-281.

DAY, R. H., \& AVERY, G. C. (1970). Absence of the horizontal-vertical illusion in haptic space. Journal of Experimental Psychology, 83, 172-173.

DAY, R. H., \& WoNG, T. S. (1971). Radial and tangential movement directions as determinants of the haptic illusion in an L figure. Journal of Experimental Psychology, 87, 19-22.

DeregowsKi, J., \& Ellis, H. D. (1972). Effect of stimulus orientation upon haptic perception of the horizontal-vertical illusion. Journal of Experimental Psychology, 95, 14-19.

Finger, F. W., \& SPELT, D. K. (1947). The illustration of the horizontal-vertical illusion. Journal of Experimental Psychology, 39, $548-551$

GREen, B. G. (1982). The perception of distance and location for dual tactile pressures. Perception \& Psychophysics, 31, 315-323.

Helson, H., \& King, S. M. (1931). The tau effect: An example of psychological relativity. Journal of Experimental Psychology, 14, $202-$ 217.

Higashiyawa, A. (1992). Anisotropic perception of visual angle: Implications for the horizontal-vertical illusion, overconstancy of size, and the moon illusion. Perception \& Psychophysics, 51, 218-230. 
Hollins, M., \& Goble, M. A. (1988). Perception of the length of voluntary movements. Somatosensory Research, 5, 335-348.

JASTROW, J. (1886). The perception of space by disparate senses. Mind, 11, 539-544.

KüNNAPAS, T. M. (1955). An analysis of the "vertical-horizontal" illusion. Journal of Experimental Psychology, 49, 134-140.

KünNAPAS, T. M. (1957). Horizontal-vertical illusion and surrounding field. Acta Psychologica, 13, 35-42.

Lederman, S. J., \& Klatzky, R. L. (1987). Hand movements: A window into haptic object perception. Cognitive Psychology, 19, 342368.

Lederman, S. J., Klatzky, R. L., Collins, A., \& Wardell, J. (1987). Exploring environments by hand or foot: Time-based heuristics for encoding distance in movement space. Journal of Experimental Psychology: Learning, Memory, \& Cognition, 13, 606-614.

LeE, D. K., MCGillis, S. L. B., \& GreensPaN, J. D. (1996). Somatotopic localization of thermal stimuli. Somatosensory \& Motor Research, 13, 67-71.

Marchetti, F. M., \& Lederman, S. J. (1983). The haptic radialtangential effect: Two tests of Wong's "moments-of-inertia" hypothesis. Bulletin of the Psychonomic Society, 21, 43-46.

Marks, L. E., \& Armstrong, L. (1996). Visual and haptic representations of space. In T. Inui \& J. L. McClelland (Eds.), Attention and performance XVI: Information integration in perception and communication (pp. 263-287). Cambridge, MA: MIT Press.

Marks, L. E., Girvin, J. P., Quest, D. O., Antunes, J. L., Ning, P., O'KeEFE, M. D., \& DobelLe, W. H. (1982). Electrocutaneous stimulation: II. The estimation of distance between two points. Perception \& Psychophysics, 32, 529-536.

MarTinez, F. (1971). Comparison of two types of tactile exploration in a task of mirror-image recognition. Psychonomic Science, 22, 124125.
ONO, A. (1969). Interdependence in successive judgments of the duration, distance and speed of a manual movement. Tohuku Psychologica Folia, 28, 29-53.

Paillard, J. (1991). Motor and representational space. In J. Paillard (Ed.), Brain and space (pp. 163-182). New York: Oxford University Press.

ReID, R. L. (1954). An illusion of movement complementary to the horizontal-vertical illusion. Quarterly Journal of Experimental Psychology, 6, 107-111.

RollmaN, G. B. (1974). Electrocutaneous stimulation. In F. A. Geldard (Ed.), Cutaneous communication systems and devices (pp. 39-51). Austin, TX: Psychonomic Society.

WAPNER, S., WeINBERG, J., GliCK, J. A., \& RAND, G. (1967). Effect of speed of movement on tactual-kinesthetic perception of extent. American Journal of Psychology, 80, 608-613.

WeBER, E. H. (1834). De pulsu, resorptione, auditu et tactu: Annotationes anatomicae et physiologicae [On pulse, breathing, hearing and touch: Anatomical and physiological writings]. Leipzig: Köhler.

WONG, T. S. (1977). Dynamic properties of radial and tangential movements as determinants of the haptic horizontal-vertical illusion with an L figure. Journal of Experimental Psychology: Human Perception \& Performance, 3, 151-164.

\section{NOTE}

1. Here and subsequently, whenever the degrees of freedom of the numerator exceeds 1, the probabilities incorporate the Huynh-Feldt correction for nonsphericity in repeated measures.

(Manuscript received February 23, 1995; revision accepted for publication June 8,1998 .) 